\title{
Tratamiento de diabetes gestacional leve: un estudio randomizado controlado, multicéntrico (1)
}

\author{
Landon MB, Spong CY, Thom E, Carpenter MW, Ramin SM, Casey B, et al. A mul- \\ ticenter, randomized trial of treatment for mild gestational diabetes. $N$ Engl $J$ Med \\ 2009;361(14):1339-48.
}

Análisis crítico: Valeria Veronesi $M^{1}{ }^{1}$, Claudio Vera $P-G, M S c^{2,3}$, Jorge Carvajal C. $P h D^{2}$

${ }^{1}$ Residente de Obstetricia y Ginecología, ${ }^{2}$ Unidad de Medicina Materno-Fetal. Departamento de Obstetricia y Ginecología, ${ }^{3}$ Unidad de Medicina Basada en Evidencias, Facultad de Medicina, Pontificia Universidad Católica de Chile.

\section{RESUMEN}

Antecedentes: Es incierto si el tratamiento de la diabetes mellitus gestacional leve mejora el resultado del embarazo. Métodos: Mujeres entre las 24 y 31 semanas de gestación y que cumplían con los criterios de diabetes mellitus gestacional leve (resultado anormal del test de tolerancia a la glucosa oral pero glicemia de ayuno menor a $95 \mathrm{mg} / \mathrm{dl}[5.3$ $\mathrm{mM} / \mathrm{l}]$ ) fueron asignadas al azar a cuidado prenatal normal (grupo control) o intervención dietaria, auto monitoreo de glicemia y terapia con insulina, si es requerida (grupo tratamiento). El resultado primario fue un compuesto de muerte fetal in útero o muerte perinatal y complicaciones neonatales, incluyendo: hiperbilirrubinemia, hipoglicemia, hiperinsulinemia y traumatismo en el parto. Resultados: Un total de 958 mujeres fueron asignadas al azar a un grupo: 458 al grupo tratamiento y 473 al grupo control. No observamos diferencias significativas entre los grupos en la frecuencia del resultado compuesto $(32,4 \%$ y $37,0 \%$ en el grupo tratamiento y control, respectivamente; $p=0,14)$. No hubo muertes perinatales. Sin embargo, hubo una reducción significativa con el tratamiento, comparado con el cuidado usual, en varios resultados secundarios pre-especificados, incluyendo peso promedio de nacimiento (3302 g vs. $3408 \mathrm{~g})$, masa grasa neonatal (5,9\% vs. $14,3 \%)$, frecuencia de niños grandes para la edad gestacional $(7,1 \%$ vs. $14,5 \%)$, peso de nacimien- to mayor de $4000 \mathrm{~g}(5,9 \%$ vs. $14,3 \%)$, distocia de hombro $(1,5 \%$ vs. $4,0 \%)$ y parto cesárea $(26,9 \%$ vs. $33,8 \%$ ). El tratamiento de la diabetes mellitus gestacional, comparado con cuidado usual, se asoció con reducción en la tasa de preeclampsia e hipertensión gestacional (tasas combinadas para las dos condiciones ( $8,6 \%$ vs. $13,6 \% ; p=0,01)$. Conclusiones. Pese a que el tratamiento de la diabetes mellitus gestacional leve no reduce significativamente la frecuencia de un resultado compuesto que incluye muerte fetal in útero o muerte perinatal y complicaciones neonatales severas, si reduce el riesgo de sobrecrecimiento fetal, distocia de hombro, parto cesárea y desordenes hipertensivos.

\section{ANÁLISIS DE LA INVESTIGACIÓN}

\section{A. Relevancia clínica de la investigación}

La pregunta qué el estudio intenta responder es: ¿Puede el tratamiento de las embarazadas con diabetes mellitus gestacional (DMG) mejorar su resultado perinatal comparado con el control obstétrico habitual (sin tratamiento específico para DMG)? Escenario clínico: La prevalencia de DMG es de 1 a $14 \%$ en Estados Unidos, variando de acuerdo a las características de la población (2). El significado clínico de la diabetes gestacional respecto al riesgo de varios resultados adversos perinatales, o a la reducción de riesgo asociado a su tratamiento es 
poco claro. Mujeres con glicemias de ayuno muy elevadas parecieran tener mayor riesgo de macrosomía y complicaciones perinatales si no reciben tratamiento, pero estas asociaciones para las formas leves son inciertas. En un estudio reciente sobre hiperglicemia y resultados perinatales adversos (HAPO) se describe fuerte asociación entre glicemia materna y aumento del peso al nacimiento, niveles de Péptido $C$ en sangre de cordón, y otros marcadores de complicaciones perinatales, incluso con glicemia bajo los niveles habituales de diagnóstico de DMG (3).

Por otro lado en países anglosajones, aun existe discusión acerca de si es necesario hacer tamizaje a la población general o sólo a población de riesgo. En Chile, sin embargo, el tamizaje para diabetes es universal en el embarazo de acuerdo con las normas nacionales vigentes, probablemente debido a la alta incidencia de DMG en nuestro país (4).

\section{B. Originalidad del estudio}

A pesar de la evidencia existente sobre el beneficio del tamizaje y tratamiento de diabetes gestacional a partir de un estudio controlado randomizado ACHOIS (5), la implementación de tamizaje para diabetes gestacional no está considerada en Estados Unidos por falta de evidencia suficiente para determinar el balance riesgo-beneficio. El estudio analizado tiene por objetivo aportar evidencia en el contexto norteamericano. En este sentido, sin ser una investigación "novedosa", si aporta información relevante para un tema no resuelto.

\section{Evaluación de la población estudiada}

Fueron seleccionables embarazadas entre $24+0$ a 30+6 semanas de gestación, con glicemias entre 135 a $200 \mathrm{mg} / \mathrm{dl}, 1$ hora después de carga de $50 \mathrm{~g}$ de glucosa. Se excluyó a las pacientes con diagnóstico previo de diabetes, historia de óbito, embarazo múltiple, asma o hipertensión arterial crónica, si estaban usando corticoides o si tenían fetos con malformaciones diagnosticadas, o con parto prematuro inminente por condiciones fetales o maternas. Todas firmaron consentimiento y el estudio fue aprobado por un comité de ética. Se sometió a las pacientes a TTGO con $100 \mathrm{~g}$ de glucosa y se incluyeron las pacientes con valores superiores a 180 $\mathrm{mg} / \mathrm{dl}$ en $1 \mathrm{hr} ; 155 \mathrm{mg} / \mathrm{dl}$ en 2 horas y $140 \mathrm{mg} / \mathrm{dl}$ en 3 horas.

\section{Intervención}

Las pacientes que cumplían los criterios fueron randomizadas por un método de urnas estratificado por centro clínico. Se dividieron en un grupo con tratamiento (consejo, auto monitorización de glicemias, dieta e insulina si lo requerían) o control obstétrico habitual. Además, de la cohorte inicial de mujeres que tenían el resultado positivo a la sobrecarga con $50 \mathrm{~g}$ de glucosa, pero que no clasificaban para diabetes gestacional en el test con $100 \mathrm{~g}$, se las incluyó en el grupo control, sin saber ni ellas ni sus tratantes esta situación, recibiendo cuidados obstétricos habituales.

Las mujeres que recibían tratamiento tenían un sistema portátil de automonitoreo de glicemia con memoria. Se indicó insulina si la mayoría de las mediciones en ayuno o $2 \mathrm{~h}$ posprandiales eran mayores a $95 \mathrm{mg} / \mathrm{dl}$ o $120 \mathrm{mg} / \mathrm{dl}$, respectivamente. Si en una paciente del grupo control existía la sospecha clínica de hiperglicemia, se debía medir glicemia sin entregar el resultado al tratante; si era $\geq 160 \mathrm{mg} / \mathrm{dl}$ o de ayuno $\geq 95 \mathrm{mg} / \mathrm{dl}$ se le informaba al investigador principal de cada centro y se iniciaba tratamiento.

\section{E. Resultados del estudio}

Desde Octubre 2002 a Noviembre 2007 un total de 1889 embarazadas fueron enroladas luego de reclasificar a pacientes con criterios de inclusión que tenían un test de tolerancia a la glucosa alterado. Las embarazadas con diabetes leve fueron asignadas a tratamiento $(n=485)$ y a control prenatal habitual $(n=473)$. Además, embarazadas con TTGO $100 \mathrm{~g}$ normal fueron incluidas en el grupo con control habitual $(n=931)$. No hubo diferencias entre los grupos de pacientes randomizadas en cuanto a variables demográficas y resultado del TTGO.

Resultados perinatales y neonatales: No hubo diferencias entre el grupo con tratamiento y el grupo control en cuanto la medida primaria de resultado, tal es un resultado compuesto que consideró: mortalidad perinatal, hipoglicemia, hiperbilirrubinemia, hiperinsulinemia neonatal y trauma al nacer. No hubo muertes perinatales. Los resultados de los componentes individuales tampoco mostraron diferencias. El promedio de peso neonatal y la grasa fetal, como también la frecuencia de grande para la edad gestacional y recién nacidos $>4000 \mathrm{~g}$, fueron significativamente inferiores en el grupo con tratamiento. La frecuencia de pequeño para la edad gestacional y admisión en unidades de cuidados intensivos neonatales no mostró diferencias. 
Tabla I

RESULTADOS PERINATALES Y NEONATALES SELECCIONADOS

\begin{tabular}{|c|c|c|c|}
\hline $\begin{array}{l}\text { Evento de interés } \\
\text { seleccionados }\end{array}$ & $\begin{array}{c}\text { RRR } \\
\text { (IC 95\%) }\end{array}$ & $\begin{array}{c}\text { RAR } \\
\text { (IC 95\%) }\end{array}$ & $\begin{array}{c}\text { NNT } \\
(\mathrm{IC95 \% )}\end{array}$ \\
\hline $\begin{array}{l}\text { Compuesto } \\
\text { primario }\end{array}$ & $\begin{array}{c}11 \% \\
(-6 \% \text { a } 28 \%)\end{array}$ & $\begin{array}{c}0,038 \\
(-0,02 \text { a } 0,097)\end{array}$ & $\begin{array}{c}26 \\
\mathrm{NNT}=10 \text { a inf } \\
\mathrm{NNH}=47 \text { a inf }\end{array}$ \\
\hline $\begin{array}{l}\text { Grandes para } \\
\text { edad gestacional }\end{array}$ & $\begin{array}{c}50 \% \\
(22 \% \text { a } 78 \%)\end{array}$ & $\begin{array}{c}0,07 \\
(0,031 \text { a } 0,109)\end{array}$ & $\begin{array}{c}14 \\
(9 \text { a 32) }\end{array}$ \\
\hline $\begin{array}{l}\text { Peso al nacer } \\
\text { mayor a } 4000 \mathrm{~g}\end{array}$ & $\begin{array}{c}58 \% \\
\text { (30\% a 85\%) }\end{array}$ & $\begin{array}{c}0,079 \\
(0,42 \text { a } 0,116)\end{array}$ & $\begin{array}{c}13 \\
(9 a 24)\end{array}$ \\
\hline
\end{tabular}

RRR: Reducción relativa de riesgo, RAR: Reducción Absoluta de Riesgo, NNT: Número necesario para tratar, NNH: Número necesario para hacer daño. Parámetros estimados con CATMAKER ${ }^{\circledR}$ de acuerdo con los datos publicados.

Resultados maternos: La tasa de inducción fue similar en los 2 grupos, sin embargo la tasa de cesárea fue mayor en el grupo sin tratamiento. Al excluir lo casos de presentación anormal, placenta previa, $\mathrm{OHA}$, y cesárea previa, la tasa de cesárea permaneció inferior en el grupo con tratamiento. El grupo con tratamiento tuvo significativamente menor tasa de distocia de hombros, preeclampsia o hipertensión gestacional. Posterior al enrolamiento, las pacientes del grupo con tratamiento tuvieron siete controles prenatales en comparación al grupo control que tuvo en promedio solo 5 , siendo esta diferencia significativa. Tanto el IMC y el aumento de peso al parto fue significativamente menor en pacientes con tratamiento. Un total de 37 mujeres en el grupo de tratamiento requirieron insulina comparado con 2 en el grupo control.

\section{F. Análisis estadístico}

El tamaño muestral fue calculado de acuerdo a la incidencia de los resultados buscados, para lograr un poder de $80 \%$ que muestre un $30 \%$ de diferencia para el evento de interés compuesto, con error tipo I de 5\%. El tamaño muestral calculado también otorga poder de más de $85 \%$ mostrando una reducción de $30 \%$ en el resultado de grande para la edad gestacional y recién nacido $>4.000$ g. El análisis estadístico se hizo primariamente de acuerdo a la intensión de tratar. Se usó un intervalo de confianza de $95 \%$ considerando los análisis interinos.

\section{G. Análisis crítico}

Validez: Los objetivos primarios y secundarios

Tabla II

RESULTADOS MATERNOS SELECCIONADOS

\begin{tabular}{|c|c|c|c|}
\hline Evento de interés & RRR (IC 95\%) & RAR (IC 95\%) & NNT (IC 95\%) \\
\hline Inducción & $\begin{array}{c}-4 \% \\
(-25 \% \text { a } 18 \%)\end{array}$ & $\begin{array}{c}-0,010 \\
(-0,066 \text { a } 0,046)\end{array}$ & $\begin{array}{c}-100 \\
\mathrm{NNT}=22 \mathrm{a} \text { inf } \\
\mathrm{NNH}=15 \mathrm{a} \text { inf }\end{array}$ \\
\hline Cesárea & $\begin{array}{c}19 \% \\
(1 \% \text { a } 37 \%)\end{array}$ & $\begin{array}{c}0,067 \\
(0,004 \text { a } 0,12)\end{array}$ & $\begin{array}{c}16 \\
(8 \mathrm{a} 230)\end{array}$ \\
\hline $\begin{array}{l}\text { Pre-eclampsia } \\
\text { o hipertensión } \\
\text { gestacional }\end{array}$ & $\begin{array}{c}35 \% \\
(6 \% \text { a } 65 \%)\end{array}$ & $\begin{array}{c}0,047 \\
(0,007 \text { a } 0,086)\end{array}$ & $\begin{array}{c}13 \\
(12 \mathrm{a} 137)\end{array}$ \\
\hline
\end{tabular}

RRR: Reducción relativa de riesgo, RAR: Reducción absoluta de riesgo, NNT: Número necesario para tratar, NNH: Número necesario para hacer daño. Parámetros estimados con CATMAKER $®$ de acuerdo con los datos publicados. 
están claramente definidos. Se utilizó un método válido de randomización (6), que siendo centralizado permite el resguardo de la secuencia. En el grupo de tratamiento los tratantes ni las pacientes fueron ciegos. En el grupo control fueron ciegos al diagnóstico tanto las pacientes como los tratantes, no se describe si los analistas de los datos fueron ciegos. El resultado primario compuesto incluye componentes de diferente importancia clínica y con una frecuencia diferente en los componentes de mayor relevancia clínica (muerte perinatal y trauma al nacer), aún así los componentes individuales no presentan diferencias significativas siendo consistentes con el resultado primario compuesto. Hubo cálculo de tamaño muestral adecuado para el resultado primario y su análisis consideró los análisis interinos pero no los múltiples análisis. El análisis de los datos se describe de acuerdo al principio de intención de tratar, pero para el resultado primario se excluyó del denominador las pacientes con pérdida de seguimiento. Las pérdidas de seguimiento, falta de datos del parto (grupo experimental $n=8$ y grupo control $n=18$ ), y pérdida de datos de laboratorio (grupo experimental $n=17$ y grupo control $n=15$ ) fueron similares en los 2 grupos, con distribución demográfica comparable entre los grupos, sin diferencias en raza o grupo étnico. Su influencia en el resultado primario es baja dado que probablemente no cambie el resultado del estudio en el peor escenario (todas las pérdidas de seguimiento con el evento de interés para el grupo de tratamiento y sin el evento de interés para el grupo control).

Comentario: El estudio analizado es de buen diseño, adecuado tamaño muestral, con una randomización adecuada que no muestra beneficio en el resultado primario y posibles beneficios en eventos de interés catalogados como secundarios.

La principal crítica está en la selección de los componentes del evento de interés primario, cuya relevancia clínica es muy disímil así como su frecuencia, con resultados ausentes en los 2 grupos para el componente más importante (mortalidad perinatal). Como conclusión no podemos decir que el tratamiento de diabetes gestacional no disminuya eventos que realmente sean relevantes para los neonatos y sus madres. Esta situación queda más clara con los eventos que son clínicamente relevantes y más frecuentes, pero que fueron considerados como eventos secundarios, como disminución de peso fetal, macrosomía, distocia de hombros, tasa de cesárea, preeclampsia e hipertensión gestacional. Lamentablemente, al ser resultados secundarios permiten, como concepto general, solo considerarlos para generar hipótesis y/o ser incluidos en meta-análisis en el contexto de la evidencia disponible, pero no son concluyentes para sustentar el tratamiento de diabetes en el embarazo en el contexto de este estudio.

Este estudio no tiene el poder suficiente para detectar diferencias significativas en resultados adversos poco frecuentes, como lesión del plexo braquial. Faltan estudios que evalúen resultados a largo plazo.

Es interesante destacar que el estudio analizado junto los estudios ACHOIS Y HAPO $(3,5)$ muestra relación de los niveles de glucosa y el riesgo de preeclampsia, con disminución de preeclampsia como beneficio del tratamiento.

Conclusión: Creemos que en este trabajo se cumple el objetivo de una evaluación rigurosa del tratamiento de DMG comparada con el control prenatal estándar en el contexto de Estados Unidos, otorgando evidencia de buena calidad que muestra ausencia de beneficio en el evento de interés perinatal compuesto propuesto, pero que muestra potenciales beneficios al disminuir el riesgo de neonato grande para la edad gestacional, la distocia de hombros, la operación cesárea y la incidencia de preeclampsia. Más estudios son necesarios para establecer en forma más precisa los beneficios del tamizaje y tratamiento de DMG en este contexto, aún así los beneficios potenciales para el neonato impresionan ser modestos.

\section{REFERENCIAS}

1. Landon MB, Spong CY, Thom E, Carpenter MW, Ramin SM, Casey B, et al. A multicenter, randomized trial of treatment for mild gestational diabetes. $N$ Engl J Med 2009;361(14):1339-48.

2. Ben-Haroush A, Yogev Y, Hod M. Epidemiology of gestational diabetes mellitus and its association with Type 2 diabetes. Diabet Med 2004;21(2):103-13.

3. Metzger BE, Lowe LP, Dyer AR, Trimble ER, Chaovarindr U, Coustan DR, et al. Hyperglycemia and adverse pregnancy outcomes. $\mathrm{N}$ Engl $\mathrm{J}$ Med 2008;358(19):1991-2002.

4. López G. Normas Técnicas Diabetes y Embarazo. En: Escobar MC, editor. Santiago: Ministerio de Salud, 1998.

5. Crowther CA, Hiller JE, Moss JR, McPhee AJ, Jeffries WS, Robinson JS. Effect of treatment of gestational diabetes mellitus on pregnancy outcomes. $\mathrm{N}$ Engl $\mathrm{J}$ Med 2005;352(24):2477-86.

6. Schulz KF, Grimes DA. Generation of allocation sequences in randomised trials: chance, not choice. Lancet 2002;359(9305):515-9. 